$>$ Les anticorps (ou immunoglobulines, Ig) produits par les lymphocytes B sont essentiels aux réponses immunitaires induites par les infections et les vaccins. Les anticorps sont des glycoprotéines hétérodimériques résultant de l'association de deux chaînes lourdes (IgH), et de deux chaînes légères ( $\mathrm{gL}$ ) d'immunoglobuline. Les chaînes IgH et IgL possèdent des régions « hypervariables », également appelées en anglais complementarity determining regions (CDR), situées dans leurs domaines variables, $\mathrm{VH}$ et $\mathrm{VL}$, qui, en se combinant, forment le site de liaison à l'antigène ou paratope. <

L'extraordinaire diversité des molécules d'anticorps provient de ces domaines variables, issus d'un réarrangement génomique aléatoire de différents gènes codant les domaines VH et VL, appelés variable «V», diversité «D» (uniquement pour les IgH) et jonction 《J 》. Ces mécanismes de recombinaison homologue $V(D) J$ s'opèrent dans les lymphocytes $B$ naissants de la moelle osseuse, et aboutissent in fine à l'expression de récepteurs Ig à la surface des lymphocytes $B$ (en anglais, $B$-cell receptors). Ces lymphocytes vont ensuite rejoindre la circulation sanguine et lymphatique, et atteindre les organes lymphoïdes secondaires dans lesquels se forment, suite à leur stimulation par les lymphocytes $T$ $\mathrm{CD}^{+}$auxiliaires en présence d'antigènes, des structures singulières appelées «centres germinatifs 》 au sein des follicules que contiennent ces organes lymphoïdes [1]. Dans les centres germinatifs, les lymphocytes B prolifèrent, opèrent une commutation de classe (ou isotype), et augmentent l'affinité de leurs Ig de surface pour l'antigène par acquisition de mutations somatiques dans les séquences de leurs domaines variables. Cette maturation de l'affinité, indispensable aux «réponses anticorps», produit les Ig de haute affinité qui sont sécrétées par les plasmocytes après leur différenciation ou exprimées à la membrane de lymphocytes $B$ mémoires [1]. De même que les plasmocytes à longue durée de vie, les lymphocytes $B$ mémoires de haute affinité patrouillant dans l'organisme contribueront à l'immunité protectrice à long terme.

\section{Ingénierie de lymphocytes B humains produisant des anticorps neutralisant le virus VIH-1 par édition génique CRISPR-Cas9}

Maxime Beretta ${ }^{1}$, Hugo Mouquet ${ }^{1}$

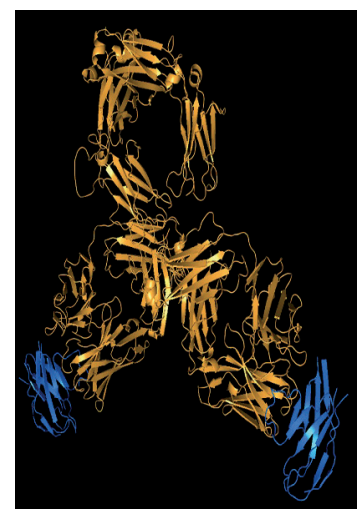

${ }^{1}$ Laboratoire d'Immunologie Humorale, Département d'Immunologie, Institut Pasteur, Inserm U1222, 28 rue du Docteur Roux, 75015 Paris, France. hmouquet@pasteur.fr

\section{Les anticorps neutralisants à large spectre, des armes préventives et thérapeutiques contre le VIH-1}

La réponse anticorps contre le virus de l'immunodéficience humaine de type 1 ( VIH-1) est inefficiente : la production d'anticorps neutralisants est trop tardive et incapable d'entraver le phénomène d'échappement viral responsable de l'émergence de variants viraux résistants. Toutefois, environ $1 \%$ des personnes infectées développent des anticorps d'une efficacité remarquable, appelés anticorps neutralisants à large spectre ou bNAb (pour broadly neutralizing antibodies), qui ciblent la protéine d'enveloppe du virus et neutralisent la majorité des quasiespèces $^{1}$ virales circulantes [2]. À partir des lymphocytes $B$ de ces individus, des dizaines de bNAb ont été clonés et produits sous forme d'anticorps monoclonaux (Figure 1), et constituent un véritable espoir pour la lutte contre le VIH-1. En effet, l'administration passive de bNAb induit une diminution considérable de la virémie chez les individus infectés et une protection très efficace contre l'infection dans les modèles animaux du VIH-1 [3]. Un vaccin protecteur devrait donc idéa-

\footnotetext{
${ }^{1}$ Les quasi-espèces correspondent aux variants viraux d'une même espèce de virus se répliquant dans un organisme et qui forment une population large et hétérogène de virions. Ce terme s'applique particulièrement aux virus à ARN tel le VIH-l qui possède un fort taux de mutations et évolue ainsi chez l'hôte qu'il infecte.
} 


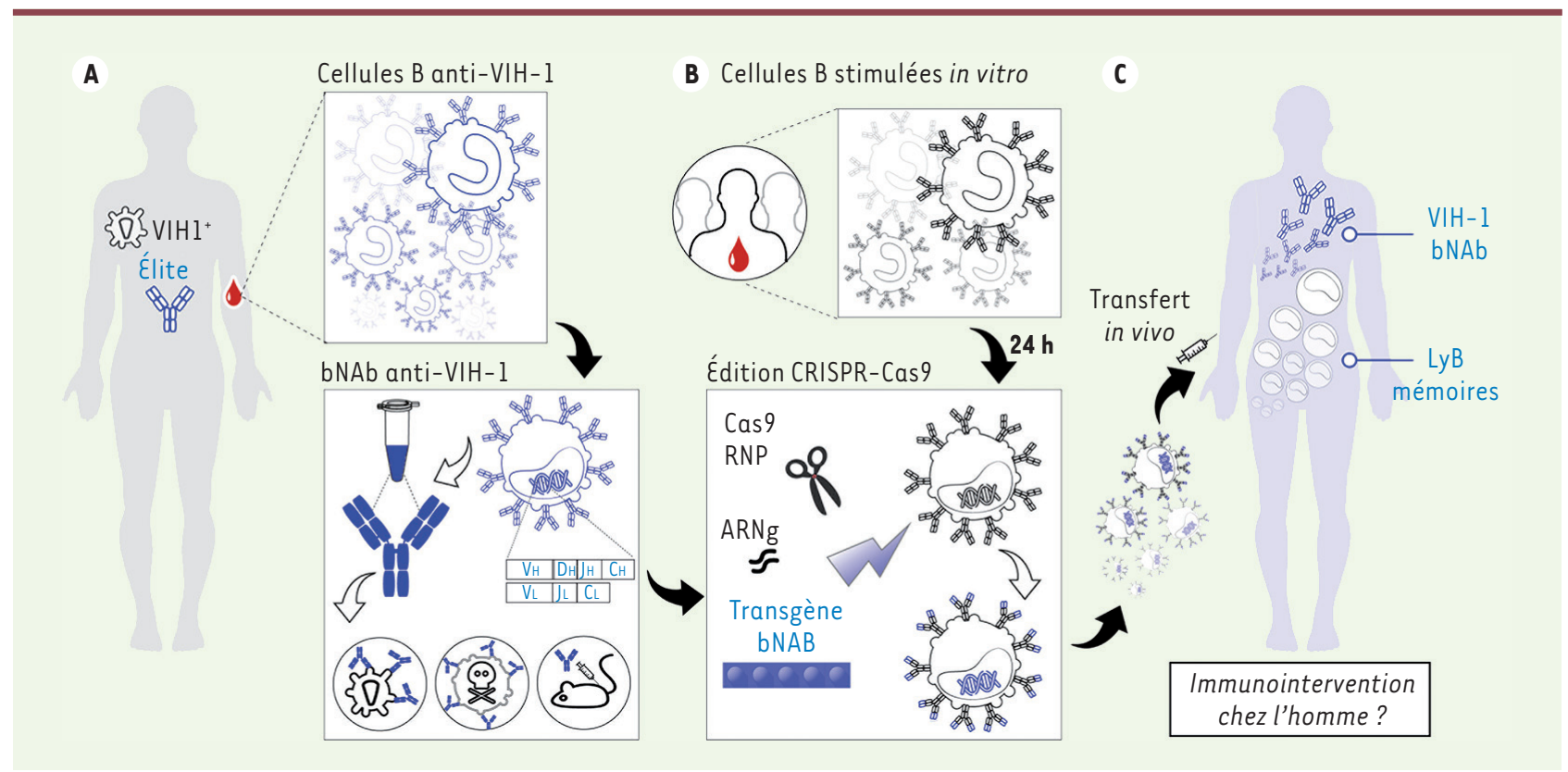

Figure 1. Stratégie d'édition génique de lymphocytes B par la technique CRISPR-Cas9 pour produire des anticorps neutralisant le virus VIH-1. A. À partir des cellules mononucléées du sang d'individus infectés par le VIH $\left(\mathrm{VIHI}^{+}\right)$et ayant développé des anticorps neutralisants à large spectre (bNAb), les lymphocytes B spécifiques des antigènes viraux sont isolés par des techniques de capture ou de criblage de cellules uniques [2]. Les gènes codant les domaines variables VH et VL des chaînes lourdes ( $\mathrm{g} \mathrm{gH}$ ) et légères ( $\mathrm{g} \mathrm{L}$ ) de ces lymphocytes $B$ anti-VIH-l sont alors amplifiés par RT-PCR, séquencés, analysés, et clonés dans des vecteurs d'expression. Les anticorps monoclonaux recombinants sont produits par transfection de cellules eucaryotes, purifiés, et caractérisés de manière très détaillée, en particulier pour leur capacité à neutraliser les différents mutants de VIH-1 et à détruire des cellules infectées in vitro et in vivo dans des modèles animaux [2]. B. Les gènes codant IgH et IgL sont alors utilisés pour construire des cassettes d'expression bNAb [12]. Grâce au système CRISPR-Cas9, ces transgènes bNAb peuvent être ensuite insérés spécifiquement dans les locus Ig de lymphocytes B humains stimulés in vitro, qui vont désormais exprimer ces bNAb d’intérêt [12]. Réimplantés in vivo, les lymphocytes $B$ transgéniques peuvent se différencier en plasmocytes qui sécrétent alors les bNAb. $C$. Théoriquement, le transfert de ces cellules productrices de bNAb chez les sujets à risque d'infection par le VIH-l permettrait de les protéger contre l'infection. Les lymphocytes B (LyB) mémoires à bNAb anti-VIH-1 présents in vivo auraient aussi l'avantage de participer aux réponses immunes contre les virus résistant au bNAb originel qui émergent chez les sujets infectés, en continuant à évoluer par maturation d'affinité et sélection de variants bNAb actifs contre ces nouveaux virus.

lement induire des anticorps de type bNAb, actifs contre la plupart des quasi-espèces de $\mathrm{VIH}-1$. En raison de phénomènes complexes de coévolution entre virus et système immunitaire à l'origine de la formation des bNAb et découverts seulement en 2013 [2], aucun vaccin reproduisant I'ontogénie naturelle des bNAb n'a encore été mis au point. Malgré cela, des progrès considérables sur les stratégies vaccinales mimant ces processus coévolutifs ont été réalisés ces cinq dernières années, et sont en faveur d'une faisabilité potentielle, à plus ou moins long terme, d'un tel vaccin chez l'homme. En l'absence de vaccin, l'une des possibilités envisagées est d'utiliser directement, chez les sujets à risque, des perfusions de bNAb à durée de vie prolongée, obtenus par ingénierie d'anticorps [4]. Cette approche prophylactique est en cours d'évaluation dans des essais cliniques chez l'homme [3]. Cependant, la facture d'anticorps de grade clinique à des fins thérapeutiques et/ou prophylactiques est assez laborieuse, chronophage, et coûteuse. Pour éviter ces contraintes, diverses stratégies de transfert et de thérapie géniques ont été explorées afin de rendre possible la production de l'anticorps d'intérêt in vivo, directement par les cellules ayant reçu le transgène d'lg. L'une de ces stratégies consiste à transduire in vivo des cellules musculaires avec des vecteurs viraux, lentivirus et virus adéno-associé, codant un anticorps neutralisant le VIH-1 [5]. Une approche ex vivo consisterait, quant à elle, à isoler des cellules souches hématopoïétiques et à les transduire avec un vecteur lentiviral codant le bNAb avant de les réimplanter in vivo [6]. Plusieurs limitations peuvent être néanmoins associées à l'utilisation de ces vecteurs, telles qu'une expression faible et non durable du transgène, ainsi que le développement d'une immunité anti-vecteur délétère. Une piste alternative prometteuse est celle reposant sur les techniques de «knock-in », qui permettent de modifier ou d' « éditer » le génome via l'induction de cassures spécifiques de I'ADN double brin par des nucléases, de type « doigts de zinc » ou TALEN (transcription activator-like effector nuclease) par exemple, puis l'intégration d'un gène exogène d'intérêt par recombinaison homologue [7]. Ce domaine de l'édition génomique a littéralement été révolutionné par la découverte du système CRISPR (clus- 
tered regularly interspaced short palindromic repeat)-Cas, identifié chez les procaryotes comme système immunitaire primitif assurant leur défense contre les bactériophages $[7,8](\rightarrow)$. L'ingénierie qui s'est développée avec l'utilisation des outils CRISPR-Cas9 permet de modifier aisément les génomes eucaryotes pour répondre $(\rightarrow)$ Voir la Synthèse de F.R. Croteau et al., $\mathrm{m} / \mathrm{s}$ $n^{\circ} 10$, octobre 2018, page 813 aux besoins d'un nombre grandissant d'applications [8], y compris celles liées à la production et l'utilisation d'anticorps monoclonaux [9-14].

\section{L'ingénierie CRISPR-Cas9 de lymphocytes B pour une production d'anticorps neutralisant le VIH-1}

En 2016, l'ingénierie immunogénomique CRISPR-Cas9 de lignées lymphocytaires $B$ a permis de commuter l'isotype des Ig endogènes à façon ou de leur faire produire uniquement leur fragment Fab (antigen-binding fragment), et, encore plus spectaculairement, de reprogrammer ces cellules pour qu'elles expriment un nouvel anticorps complet d'origine « exogène » $[10,14]$. Un niveau supérieur a été franchi cette année par l'édition CRISPR-Cas9 de lymphocytes B humains primaires, permettant la génération de lymphocytes $B$ transgéniques capables de produire des anticorps neutralisants à visée thérapeutique, en particulier contre le VIH-1 [9, 11-13]. L'étude la plus élégante et aboutie a été réalisée par l'équipe du Pr Michel Nussenzweig de l'Université Rockefeller à New York, qui, grâce à la technique CRISPR-Cas9, a réussi à produire des lymphocytes $B$ humains exprimant des bNAb anti-VIH-1 prometteurs pour une éventuelle application médicale [3] (Figure 1). Les auteurs de cette étude ont tout d'abord procédé à l'inactivation des locus d'Ig endogènes, car l'insertion d'un transgène anticorps peut conduire à des combinaisons inappropriées des $\operatorname{lgH}$ et $\mathrm{IgL}$ endogènes et exogènes produites par ces lymphocytes $B$ génétiquement modifiés. La chaîne IgL endogène est simplement rendue non fonctionnelle par l'utilisation de guides ARN-CRISPR spécifiques d'un exon du gène codant cette chaîne, suivie de son clivage par la nucléase Cas9. L'inactivation de la chaîne IgH se fait directement du fait de l'insertion, par le système CRISPR-Cas 9 , du transgène bNAb dans la région intronique du gène codant cette chaîne, à une centaine de paires de bases en aval du dernier segment $J_{H}$. L'insertion de la construction transgénique ne modifiant pas les éléments naturels de régulation génique et d'épissage des transcrits du gène codant IgH, les formes membranaire et sécrétée des anticorps sous différents isotypes peuvent être exprimées. Dans cette étude, l'édition CRISPR-Cas9 de lymphocytes B humains isolés de donneurs sains pour produire deux bNAb anti-VIH-l d'intérêt clinique [3] s'est avérée très efficace. L'un des avantages majeurs de l'édition immunogénomique de lymphocytes $B$ est de conserver les mécanismes biologiques des réponses immunitaires impliquant les lymphocytes B tout en leurs conférant une nouvelle spécificité anticorps, d'intérêt anti-infectieux par exemple et, dans ce cas précis, anti-VIH-1. Théoriquement, ces lymphocytes B transgéniques peuvent participer activement à l'immunité contre le VIH-l en sécrétant des anticorps neutralisants, mais aussi en continuant à évoluer face à l'émergence de virus devenus résistants au bNAb d'origine
(Figure 1). Les travaux de l'équipe du Pr Nussenzweig confirment en partie cette hypothèse dans un modèle murin où les lymphocytes $B$ murins édités pour exprimer les bNAb sont capables de répondre in vivo à l'immunisation contre un antigène d'enveloppe du VIH-1. Les lymphocytes $B$ reprogrammés répondent globalement de manière tout à fait classique, en sécrétant les anticorps humains bNAb neutralisant le VIH-1, détectés dans le sérum des animaux. La question de l'évolution de ces lymphocytes $B$ dans les centres germinatifs via la sélection de mutations somatiques dans les domaines variables $\mathrm{VH}$ et $\mathrm{VL}$ en réponse à l'échappement du virus par mutation n'est pas abordée dans cette étude, mais une telle évolution apparaît très plausible au regard de travaux antérieurs utilisant une approche similaire [9].

\section{Vers une édition immunogénomique CRISPR-Cas9 de lymphocytes B antiviraux chez l'homme?}

En l'absence de vaccins contre certains agents infectieux tels que le virus respiratoire syncytial, responsable des infections respiratoires les plus fréquentes chez les jeunes enfants, ou le VIH-1, l'édition génomique CRISPRCas9 de lymphocytes $B$ humains exprimant de puissants anticorps humains neutralisants représente une alternative très prometteuse dans la prévention de maladies infectieuses [9, 11, 12]. Cette approche permettrait aussi de pallier la nécessité d'injecter de façon itérative les anticorps monoclonaux lors d'interventions thérapeutiques chez les sujets déjà infectés, en leur assurant une immunité à long terme. Dans les deux modalités, prophylactique et thérapeutique, les lymphocytes $B$ édités exprimant à leur surface des bNAb ont l'avantage, par rapport à un anticorps monoclonal, d'avoir un potentiel de maturation et d'évolution en réponse aux virus qui se diversifient, tels que ceux de la grippe et du $\mathrm{VIH}-1$. Bien qu'induire la production de bNAb anti-VIH-l par vaccination soit désormais envisageable, développer un vaccin universel efficace contre l'ensemble des variants viraux reste un défi majeur pour la communauté scientifique. La possibilité d'utiliser cette technique d'édition avec succès chez les individus séropositifs ou à fort risque d'infection reste cependant à l'heure actuelle incertaine. Davantage d'études in vivo sont requises, en particulier dans les modèles de primates non-humains qui miment le mieux l'infection, afin d'assurer la faisabilité de cette approche chez l'homme. De futures expérimentations contribueront certainement à optimiser l'efficacité et à accroître la durée des réponses anticorps dues aux lymphocytes B reprogrammés avec des spécificités bNAb complémentaires, permettant des combinaisons d'anticorps très efficaces. Elles devront 
également contrôler l'absence d'intégration des transgènes au hasard dans le génome (en anglais, off-targets), qui est potentiellement délétère et dangereuse. L'ingénierie CRISPR-Cas9 de lymphocytes B humains afin de produire des anticorps neutralisants est un nouvel outil indubitablement essentiel au développement des stratégies d'édition génique pour combattre l'infection par le VIH-1 [5]. On peut donc s'attendre à ce qu'un nombre important d'essais cliniques fondés sur cette technique soient menés au cours de la prochaine décennie. $\diamond$

\section{CRISPR-Cas9 editing of HIV-1 neutralizing human B cells}

\section{LIENS D'INTÉRÊT}

Les auteurs déclarent n'avoir aucun lien d'intérêt concernant les données publiées dans cet article.

\section{RÉFÉRENCES}

1. Victora GD, Nussenzweig MC. Germinal centers. Annu Rev Immunol $2012 ; 30: 429-57$.

2. Mouquet H. Antibody B cell responses in HIV-1 infection. Trends Immunol $2014 ; 35$ : 549-61.

3. Gruell H, Klein F. Antibody-mediated prevention and treatment of HIV-1 infection. Retrovirol $2018 ; 15: 73$

4. Gautam R, Nishimura Y, Gaughan N, et al. A single injection of crystallizable fragment domainmodified antibodies elicits durable protection from SHIV infection. Nat Med $2018 ; 24: 610-6$.
5. Falkenhagen A, Joshi S. Genetic strategies for HIV treatment and prevention. Mol Ther Nucleic Acids $2018 ; 13: 514-33$.

6. Kuhlmann AS, Haworth KG, Barber-Axthelm IM, et al. Long-term persistence of anti-HIV broadly neutralizing antibody-secreting hematopoietic cells in humanized mice. Mol Ther $2019 ; 27: 164-77$.

7. Gaj T, Gersbach CA, Barbas CF. ZFN, TALEN, and CRISPR/Cas-based methods for genome engineering. Trends Biotechnol 2013 ; 31 : 397-405.

8. Croteau FR, Rousseau GM, Moineau S. Le système CRISPR-Cas : au-delà de l'édition génomique. Med Sci (paris) 2018 ; $34: 813-9$.

9. Voss JE, Gonzalez-Martin A, Andrabi R, et al. Reprogramming the antigen specificity of B cells using genome-editing technologies. elife 2019; 8 : e42995.

10. Pogson M, Parola C, Kelton WJ, et al. Immunogenomic engineering of a plugand-(dis)play hybridoma platform. Nat Commun $2016 ; 7: 12535$.

11. Moffett HF, Harms CK, Fitzpatrick KS, et al. B cells engineered to express pathogen-specific antibodies protect against infection. Science Immunol 2019 ; 4 : eaax0644.

12. Hartweger $H$, McGuire AT, Horning M, et al. HIV-specific humoral immune responses by CRISPR-Cas9-edited B cells. J Exp Med 2019; 216 : 1301-10.

13. Greiner V, Bou Puerto R, Liu S, et al. CRISPR-mediated editing of the B cell receptor in primary human $B$ cells. iScience $2019 ; 12: 369-78$.

14. Cheong T-C, Compagno M, Chiarle R. Editing of mouse and human immunoglobulin genes by CRISPR-Cas9 system. Nat Commun 2016 ; 7 : 10934.

\section{TIRÉS À PART}

H. Mouquet

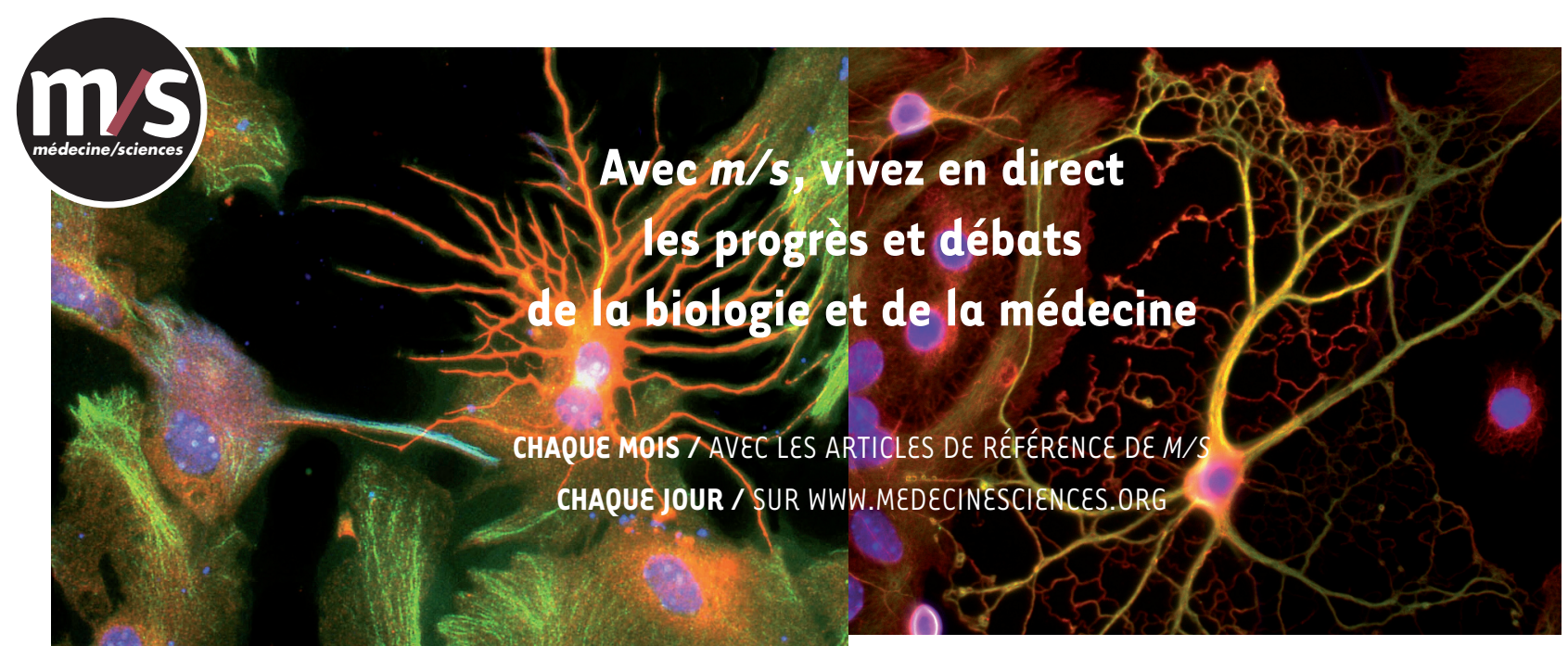

Abonnez-vous sur

www.medecinesciences.org

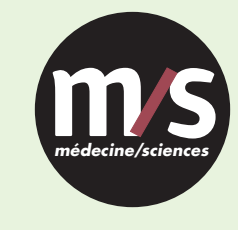

Tarifs d'abonnement m/s - 2019

Abonnez-vous

à médecine/sciences
$>$ Grâce à $m / s$, vivez en direct les progrès des sciences biologiques et médicales

Bulletin d'abonnement page 1226 dans ce numéro de $\mathrm{m} / \mathrm{s}$

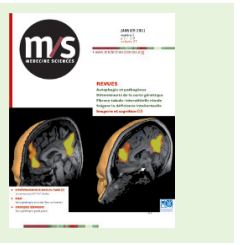

